\title{
On identifiability in the autopsy model of reliability systems of lifemonitored and conditionally lifemonitored components
}

\author{
Jørund Gåsemyr, \\ Univ. of Oslo
}

\begin{abstract}
The autopsy data of a coherent system is the pair $(T, I)$, where $T$ is the time to failure of the system, and $I$ is the set of components failed by that time. A basic question is under which conditions the distribution of the autopsy data determines the simultaneous life distribution of the components. This problem has been studied under various distributional and structural assumptions (always under the assumption of independent component life distributions) by Meilijson (1981), Nowik (1990) and Antoine et al.(1993). In this paper we study the same problem in the situation when the autopsy data is supplemented with data from lifemonitoring and conditional lifemonitoring of some components, as introduced by Meilijson (1994).
\end{abstract}

\section{Introduction}

Let $(E, \phi)$ be a coherent system, with components $E=\{1, \ldots, n\}$. The state of the $i$ th component at time $t$ is denoted by $X_{i}(t)\left(X_{i}(t)=1\right.$ if the $i$ th component functions, $X_{i}(t)=0$ otherwise). The state of the system is given by $\phi\left(X_{1}(t), \ldots, X_{n}(t)\right)=\phi(\mathbf{X}(t))=1$ if the system functions, and 0 otherwise. Define $T_{i}=\inf \left\{t \mid X_{i}(t)=0\right\}=$ the lifetime of the $i$ th component. Failed components are not repaired, so $X_{i}(t)=0$ if $t \geq T_{i}$. The $T_{i}$ 's are assumed to be independent with distribution functions $F_{i}, i=1, \ldots, n$. The lifetime of the system is given by $T=\inf \{t \mid \phi(\mathbf{X}(t))=0\}$. Denote by $I$ the set of components that have failed by time $T$, i.e. $I=\left\{i \mid T_{i} \leq T\right\}$. $I$ is the socalled diagnostic set, and the pair $(T, I)$ is called the autopsy data of the system. The autopsy data arise when the system is observed from time 0 until system failure, after which it is examined to find out which components have failed and which have survived.

The question of identifiability of the system, i.e. whether the distribution of the autopsy data determines the life distributions of the components, has been addressed by Meilijson (1981), Nowik (1990) and Antoine et al. (1993). The latter paper contains an excellent introduction, motivating the problem 
and summarising the main results. We give here a short review of the basic definitions and results needed in the present paper.

The incidence vector of a subset $A$ of $E$ is the vector $(I(1 \in A), \ldots, I(n \in$ $A)$ ). A cut set of $(E, \phi)$ is a subset $K \subset E$ such that the incidence vector $\mathbf{x}$ of $K$ satisfies $\phi(\mathbf{1}-\mathbf{x})=0$. A minimal cut set is a cut set with no other cut set as a proper subset. A fatal set of $(E, \phi)$ is a subset $A \subset E$ such that the intersection of all minimal cut sets contained in $A$ is nonempty, and this intersection is called the critical set of $A$ and is denoted by $C_{A}$. Under quite general conditions, and in particular, if $F_{i}$ is continuous and strictly increasing for each $i, A$ is fatal if and only if $P(I=A)>0$, and $i \in C_{A}$ if and only if $P\left((I=A) \cap\left(T_{i}=T\right)\right)>0$. We denote by $\mathcal{A}=\left\{A_{1}, \ldots, A_{m}\right\}$ the set of fatal sets, and by $M, D, C$ the minimal, fatal and critical incidence matrices respectively; i.e. the matrices whose rows consist of incidence vectors of the minimal cut sets, the fatal sets and the corresponding critical sets respectively. If $B$ is any of these matrices, and $A$ is a subset of $E$, we denote by $B_{A}$ the submatrix consisting of the columns corresponding to the indices in $A$.

The distribution of the autopsy data is defined through the equations

$$
\begin{aligned}
& P\left((T \leq t) \cap\left(I=A_{i}\right)\right)=G_{i}(t)= \\
& \quad=\int_{0}^{t} \prod_{j \in A_{i}-C_{A_{i}}} F_{j}(s) \prod_{j \in A_{i}^{c}} \bar{F}_{j}(s) d\left(\prod_{j \in C_{A_{i}}} F_{j}(s)\right)
\end{aligned}
$$

where $\bar{F}_{j}(t)=1-F_{j}(t)$. The question of identifiability of the system reduces to the question of whether these equations determine $F_{1}, \ldots, F_{n}$ as functions of $G_{1}, \ldots, G_{m}$. For some components the problem is easy, as pointed out by Nowik (1990). Define

$$
\begin{aligned}
J= & \{i \mid \text { there exist } j, k \in\{1, \ldots, m\} \text { such that } \\
& \left.i \in A_{j}, A_{k}=A_{j}-\{i\} \text { and } C_{A_{j}}=C_{A_{k}}\right\}
\end{aligned}
$$

If $i \in J$, with $i, j, k$ satisfying (2), we clearly have from (1)

$$
\left(d G_{j}(t)\right) /\left(d G_{k}(t)\right)=\left(F_{i}(t)\right) /\left(\bar{F}_{i}(t)\right)
$$

Thus, the components in $J$ are trivially identifiable. As an illustration, suppose that $(E, \phi)$ consists of two modules in series. Thus, $E$ is the disjoint union of subsets $E_{1}, E_{2}$, and $\phi(\mathbf{x})=\phi\left(\mathbf{1}_{E_{2}}, \mathbf{x}\right) \phi\left(\mathbf{1}_{E_{1}}, \mathbf{x}\right)$, where $\left(\mathbf{1}_{E_{l}}, \mathbf{x}\right)$ denotes the vector whose coordinates are 1 corresponding to $E_{l}, l=1,2$, and coincide with $\mathbf{x}$ otherwise. If $i \in E_{1}$ is not in series with the rest of the system, and $K_{2}$ is a minimal cut set contained in $E_{2}$, the definition is satisfied with $A_{k}=K_{2}$ and $A_{j}=K_{2} \cup\{i\}$, so the $i$ th component is identifiable. 
Combining this fact with the fact that any series system is identifiable (which follows from the main result of Meilijson (1981), see below), it is easy to see that $(E, \phi)$ is identifiable. This generalises proposition 4 of Antoine et al. (1993), which assumes analytic distribution functions.

Meilijson (1981) considers the reduced system consisting of the equations (1) corresponding to minimal cut sets (which are of course also fatal sets). His main result is that the system is identifiable if $M$ has full rank (i.e. rank $\mathrm{n})$, provided that the $F_{i}$ 's are continuous with the same essential extrema. Hence, a series system is identifiable if the distributional conditions are satisfied, $M$ being the identity matrix. Nowik (1990) shows that if $C_{J c}$ has full rank, and if the $F_{i}$ 's are mutually absolutely continuous with common extrema, and with positive atoms at the common infimum, the system is identifiable. The rank condition is shown to be equivalent to the condition that at most one component is in parallel with the rest of the system. This latter condition is shown by Antoine et al. (1993) (theorem 2) to be equivalent to the condition that $D$ has full rank. An application of this rank result is that any $k$-out-of- $n$ system with $k>1$ is identifiable (the system functions if at least $k$ components are functioning). Indeed, $D$ has full rank since $k \neq 1$, and we have $M=D$ since each fatal set is a minimal cut set. Identifiability then follows from Meilijson's theorem (this result is proved directly in Meilijson (1981)). The rank condition is clearly necessary for identifiability, no matter what assumptions that are made on the distributions. Based on computational studies, Antoine et al. (1993) conjectured that it is also sufficient (at least when the distribution functions are analytic), but were not able to prove this. They give, however, a stronger sufficient condition, assuming that each $F_{i}$ is analytic, with $r_{i}$ the smallest power in the power series expansion, and $a_{i 0}$ the corresponding coefficient (theorem 4 of Antoine et al. (1993)). From the proof of this theorem it follows that if $D$ has full rank, then $r_{i}$ and $a_{i 0}$ are determined for each $i$, covering many important parametric classes of distributions. The theorem states that the system is identifiable if the matrix $R D+p C$ has full rank for $p=1,2, \ldots$ and all possible choices of $R$, where $R$ denotes the diagonal matrix whose $i$ th diagonal entry is $r_{1} c_{i 1}+\cdots+r_{n} c_{i n}$, where $c_{i j}$ are the entries of $C$.

In the present paper we study identifiability in a more general model, where the autopsy data is supplemented with data from partial observation of specific components. Components are monitored according to a particular observation plan, specifying a set of lifemonitored components (i.e. components $i$ that are monitored from time 0 until $T_{i} \wedge T$ ), and a set of conditionally monitored components (i.e. components $i$ that are monitored from an inspection time $\tau_{i}$ determined by the observed history, or, to be more precise, the 
history that would have been observed, had there been no observation of the system failure time $T$, and had not observation of the system been censored at $T)$. We require $P\left(\tau_{i} \leq T\right)>0$ for the $i$ th component to be considered as conditionally monitored. The concepts of lifemonitored and conditionally monitored components were introduced in Meilijson (1994), where algorithms for maximum likelihood estimation of the $F_{i}$ 's are discussed (assuming parametric distributions). In Gåsemyr and Natvig (1994) the exact likelihood function for such data is derived, making Baysian estimation of the life distributions possible. Precise definitions are given in section 2, where we define two types of observation plans, differing with respect to the way the inspection times $\tau_{i}$ arise. We denote by $R_{0}$ and $M_{0}$ the set of lifemonitored and conditionally lifemonitored components respectively. We denote by $\mathbf{D}$ the data arising from the observation plan, and by $F$ the distribution of this data. $\mathbf{D}$ consists of the following variables:

(i) $\left(T_{i} \wedge T, I\left(T_{i} \leq T\right)\right.$ for $i \in R_{0}$

(ii) $\left(\tau_{i}, I\left(T_{i} \leq \tau_{i}\right)\right.$ if $\tau_{i} \leq T, i \in M_{0}$

(iii) $\left(T_{i} \wedge T, I\left(T_{i} \leq T\right)\right.$ if $\tau_{i} \leq T_{i} \wedge T, i \in M_{0}$

(iv) The autopsy data $(T, I)$

We show in section 2 that under the same assumptions as in the main theorem of Meilijson (1981) the life distributions of all lifemonitored components are identifiable. Assuming, in addition, that the distribution functions are strictly increasing, the life distributions of the conditionally monitored components are shown to be identifiable under certain assumptions on the observation plan. The reason we have to make such assumptions, is the dependence of $T_{i}$ on the event $\tau_{i}<T$ for a conditionally monitored component $i$. The assumptions we make, are related to a condition in Gåsemyr and Natvig (1994) which greatly simplifies the likelihood expression. In section 3 we specialise to analytic distribution functions and relate the results of section 2 to the results of Antoine et al. (1993).

\section{Identifiability of lifemonitored and condi- tionally lifemonitored components}

Throughout this section, assume that the component life distributions are continuous and have common essential extrema. Not surprisingly, the case of lifemonitored components is quite straightforward: 
In the second type of observation plan several "inspection rounds" may be performed at each observed component failure time $Z_{k}$.

Definition 3 (Type 2 observation plan (several inspection rounds)) $P u t Q_{0}=\emptyset$. Suppose that $R_{s}, Q_{s}$ and $K(s)$ are defined for $s \leq l$. Suppose also $Z_{r}$ is defined for $r \leq k=K(l)$. If $R_{l}=\emptyset$, the observation plan is terminated. Otherwise, we have two separate cases.

Case 1: $H\left(R_{l}, Q_{l}\right) \neq \emptyset$. Define $H_{l+1}=H\left(R_{l}, Q_{l}\right) K(l+1)=K(l)=k$ $\tau_{j}=Z_{k}$ for $j \in H_{l+1} Y_{j}=X_{j}\left(\tau_{j}\right), j \in H_{l+1} \quad R_{l+1}=R_{l} \cup\left\{j \in H_{l+1} \mid Y_{j}=1\right\}$ $Q_{l+1}=Q_{l} \cup\left\{j \in H_{l+1} \mid Y_{j}=0\right\}$

Case 2: $H\left(R_{l}, Q_{l}\right)=\emptyset$. Define $K(l+1)=K(l)+1=k+1 Z_{k+1}=$ $\min \left\{T_{i} \mid i \in R_{l}\right\} \quad I_{k+1}=i$ if $T_{i}=Z_{k+1} H_{l+1}=H\left(R_{l}-\left\{I_{k+1}\right\}, Q_{l} \cup\left\{I_{k+1}\right\}\right)$ $\tau_{j}, Y_{j}$ as in case $1 R_{l+1}=\left(R_{l}-\left\{I_{k+1}\right\}\right) \cup\left\{j \in H_{l+1} \mid Y_{j}=1\right\} Q_{l+1}=$ $Q_{l} \cup\left\{I_{k+1}\right\} \cup\left\{j \in H_{l+1} \mid Y_{j}=0\right\}$. We then say that the inspection times $\tau_{j}$ define an observation plan of type 2.

The type 2 observation plan clearly gives greater control over the risk set (the set of monitored components). For both plans it is assumed that the inspection procedure and installation of monitoring equipment takes 0 time (time may be taken as operational time). It does not follow from the definitions that $P\left(\tau_{j} \leq T\right)>0$ for each $j \in M_{0}$, but if this is not the case, $M_{0}$ may be redefined.

The observed history arising from an observation plan contains information about $T$. For any component $i, T_{i}$ and $T$ are dependent, so $T_{i}$ is stochastically dependent on the observed history in general. In particular, if component $i$ is conditionally monitored, $T_{i}$ is dependent on the history prior to $\tau_{i}$. For any such history satisfying $\tau_{i} \leq T, F$ determines the conditional distribution of $T_{i}$ given the history, but this may differ from the unconditional distribution in general. In the following proposition, conditions are given, in terms of the existence of certain types of histories, that eliminate this dependence problem. The event $A$ appearing in the proposition, represents (part of) such a history prior to $\tau_{i}$ (the clue being the choice $i \varepsilon H_{l+1}-H$ ). Special cases are given in the subsequent corollaries.

Proposition 1 Suppose $F_{p}$ is continuous and strictly increasing for each $p \in E$. Assume $i \in M_{0}$. Suppose there exists an event $A$ of one of the following two forms:

(i) $A=\left(\cap_{s=1}^{l}\left(I_{s}=i_{s}\right) \cap\left(\cap_{m \in H_{s}}\left(Y_{m}=y_{m}\right)\right)\right) \cap\left(\left(I_{l+1}=j\right) \cap\left(\cap_{m \in H}\left(Y_{m}=\right.\right.\right.$ $\left.\left.\left.y_{m}\right)\right)\right)$, with $H \subset H_{l+1}$, arising from an observation plan of type 1 
(ii) $A=\left(\cap_{s=1}^{l}\left(\cap_{m \in H_{s}}\left(Y_{m}=y_{m}\right)\right)\right) \cap\left(\cap_{r=1}^{K(l+1)}\left(I_{r}=i_{r}\right)\right) \cap\left(\cap_{m \in H}\left(Y_{m}=y_{m}\right)\right)$, where $H \subset H_{l+1}$, and $i_{K(l+1)}=j$, arising from an observation plan of type 2

satisfying $P(A)>0$, such that $i \in H_{l+1}-H$, and such that one of the following two conditions is satisfied:

(a) $A$ implies $T \geq T_{j}$

(b) $A \cap\left(T_{i} \geq T_{j}\right)$ implies $T \geq T_{j}$, and $F_{p}$ is determined by $F$ for all $p$ such that the pth component is involved in the definition of $A$

Then $F_{i}$ is determined by $F$.

Proof: We first consider part (a). If $A$ is of the form (i) or (ii), put $A(t, d t)=$ $A \cap\left(t-d t<T_{j}<t\right)$. Since all $F_{p}$ 's $(p \in E)$ are strictly increasing, $P(A(t, d t))>0$ for all $t$ in the common essential support, and for all $d t>0$. Moreover, $A(t, d t)$ is observed if it occurs, since $A$ implies $T \geq T_{j}$. Hence, $P(A(t, d t))$ is determined by $F$. Obviously, the event $A(t, d t)) \cap\left(T_{i}>T_{j}\right)$ also implies $T \geq T_{j}$, so the probability of this event is determined by $F$ as well. Hence $F$ determines $P\left(T_{i}>T_{j} \mid A(t, d t)\right)$. But we have $P\left(T_{i}>T_{j} \mid A(t, d t)\right)=$ $P\left(T_{i}>t \mid A(t, d t)\right)+P\left(t-d t<T_{j}<T_{i} \leq t \mid A(t, d t)\right)$. Since $\left.A(t, d t)\right)$ is defined by $T_{p}$ 's for $p \neq i, T_{i}$ is independent of $A(t, d t)$, and the first summand is $P\left(T_{i}>t\right)=\bar{F}_{i}(t)$. The second summand is less than $P\left(t-d t<T_{i} \leq t\right.$ ) (using independence again), which approaches 0 as $d t$ tends to 0 by the continuity of $F_{i}$. This proves part (a). Part (b) is proved similarly, the only difference being that $P(A(t, d t))$ is determined by $F$ because of the identifiability of the components defining $A$.

The crucial condition $A$ implies $T \geq T_{j}$ is obtained for a very interesting type of inspection function.

Definition 4 The inspection function $H$ is said to be cause controlling if

(i) whenever $R \cup\left(M_{0}-Q\right)$ is a path set (i.e. a set intersecting each cut set), we also have that $R \cup H(R, Q)$ is a path set.

(ii) If $R \cup\left(M_{0}-Q\right)$ is not a path set, then $H(R, Q)=M_{0}-(R \cup Q)$.

If such an inspection function is applied in an observation plan of type 2 and $R_{0}$ is chosen as a path set as well, the set of monitored components will always be sufficiently large to ensure that the system is functioning, as long as this is possible. The name is motivated by the fact that the component failing at the same time as the system (i.e. the immediate cause of system failure) is always identified, provided it belongs to $R_{0} \cup M_{0}$. If $H$ is applied in 
an inspection plan of type 1, these statements are true in the event that all inspected components are found to be functioning at the time of inspection; an event which has positive probability. A special case of a cause controlling inspection function is the case when $H(R, Q) \cup R$ is a path set unless $Q$ is a cut set (which follows from the definition if $R_{0} \cup M_{0}=E$ ), which is shown in corollary 5.4 in Gåsemyr and Natvig (1994) to give a very simple likelihood function compared to the general case.

Corollary 1 Suppose an observation plan of type 1 or 2 , with $R_{0}$ chosen as a path set, is determined by a cause controlling inspection function $H$, and that the $F_{p}$ 's are continuous and strictly increasing for each $p \in E$. Then $F_{i}$ is determined by $F$ for each $i \in M_{0}$.

Proof: We only consider the type 1 observation plan, the type 2 can be treated almost identically. Let $\left\{B_{l}\right\}, l=1, \ldots, L$ be a sequence of events arising from the observation plan until at $Z_{L}$ all components in $M_{0}$ have been inspected, of the form $B_{l}=\cap_{s=1}^{l}\left(\left(I_{s}=i_{s}\right) \cap\left(\cap_{m \in H_{s}}\left(Y_{m}=1\right)\right)\right)$. Thus, $B_{l}=\cap_{s=1}^{l} B_{s}, l=1, \ldots, L$, and $P\left(B_{L}\right)>0$. Since $H$ is cause controlling, it is seen that $B_{l}$ implies $T \geq Z_{l+1}$ for each $l \leq L-1$, and also that $i \in H_{l}$ for some $l \leq L$. By taking $A$ as the event $B_{l-1} \cap\left(I_{l}=i_{l}\right)$, the corollary follows from proposition 1 , part (a).

Note that at least two components in parallel with the rest of the system are definitely not identifiable from pure autopsy data, since any component in parallel with the rest of the system belongs to every fatal set, and the autopsy data therefore necessarily contains exactly the same information on all such components. Therefore, the following corollary is a particularly interesting application of proposition 1 and corollary 1 . The observation strategy in part (a) is to sequentially monitor the components that are in parallel with the rest of the system, one at a time.

Corollary 2 Suppose each $F_{p}$ is continuous and strictly increasing. Let $P=$ $\{1,2, \ldots, k\}$ be the components that are in parallel with the rest of the system, i.e. $P$ is contained in every cut set, and put $S=E-P$.

(a) Define $R_{0}=\{1\}, H(\emptyset,\{1, \ldots, j\})=\{j+1\}, j=1, \ldots, k-1$. (H may be left undefined for other values of $R, Q)$. Then $F_{i}$ is determined by $F$ for each $i \in P$.

(b) Suppose that $P \subset R_{0} \cup M_{0}$, and that $F_{p}$ is determined by $F$ for each $p \in S \cap M_{0}$. Then $F_{i}$ is determined by $F$ for each $i \in P$. If in addition $F_{p}$ is determined by $F$ for each $p \in S-\left(M_{0} \cup R_{0}\right)$, then $(E, \phi)$ is identifiable. 
Proof: Part (a) follows from corollary 1 , since clearly $R_{0}=\{1\}$ is a path set and $H$ is cause controlling (alternatively, part (a) may be considered as a special case of part (b)). To prove part (b), define the ordered inspection times $\tau_{(1)}<\tau_{(2)}<\ldots$. Choose $i \in P$. If $i \in R_{0}$, the identifiability of $F_{i}$ follows from theorem 1. Suppose that $i \in M_{0}$ and $P\left(\tau_{i}=\tau_{(1)}\right)>0$. This implies that there exists an event $A$ as in proposition 1, defined only in terms of components whose life distributions are determined by $F$ (in fact, components in $R_{0}$ ), with $T_{j}$ denoting the last observed failure time, such that $\tau_{i}=T_{j}$. Since $T_{i} \geq T_{j}$ then implies $T \geq T_{j}$, the identifiability of $F_{i}$ follows from proposition 1, part (b). To prove the result for arbitrary $i$, we may proceed by induction on the smallest integer $r$ such that $P\left(\tau_{i}=\tau_{(r)}\right)>0$.

Remark 1 In the verification of the hypothesis of identifiability of $F_{p}$ for $p \in S$ in part (b) of corollary 2, the other results of this section may be helpful. Note also that if at least one component is in parallel with the rest of the system, every cut set is a fatal set. This makes it potentially easy to identify components as members of $J$. For instance, suppose that $i \in S$ does not belong to any path set of length 2 contained in $S$. Then the coherent structure function $\phi\left(1_{i}, \mathbf{0}_{P}, \mathbf{x}\right)$, defined on the components in $S-\{i\}$, defines a system with no components in parallel with the rest of the system, and hence with no components in the intersection of all minimal cut sets. It follows that the fatal sets $E$ and $E-\{i\}$ of the original system have the same critical set, namely $P$, and hence that $i \in J$.

Remark 2 A slightly different version of corollary 2 part (b) can be given. Assuming $F_{p}$ is identifiable for all $p \in S$, we may allow one component in $P$, say component 1 , to be left out from $R_{0} \cup M_{0}$, provided $P\left(\cap_{l \in M_{0} \cap P}\left(\tau_{l} \leq T\right)\right)>$ 0 . Then the observation plan may, with positive probability, result in an event of the form $B_{1}=\cap_{s=1}^{l}\left(\left(I_{s}=i_{s}\right) \cap\left(\cap_{m \in H_{s}}\left(Y_{m}=y_{m}\right)\right)\right) \cap\left(i_{l} \in P-\{1\}\right) \cap$ the observation plan is terminated at $\left.T_{i_{l}}\right)$ with $P-\{1\} \subset R_{0} \cup H_{0}$, where $H_{0}$ denotes the set $\left(\cup_{s=1}^{l} H_{s}\right)$, the set of components that have been inspected. $B_{1}$ is observed if it occurs, and therefore $F$ determines the probability $G(t)$ of the event $B_{1} \cap\left(T_{i_{l}}<t\right)$. Likewise, the event $B_{2}=B_{1} \cap\left(T_{i_{l}}<T\right) \cap(I=E)$ has positive probability and is observed if it occurs, so that $F$ determines $G_{0}(t)=P\left(B_{2} \cap(T \leq t)\right)$. But on $B_{2}$ we must necessarily have $T=T_{1}$, and hence

$$
G_{0}(t)=\int_{0}^{t} G(s) \prod_{j \in S-\left(R_{0} \cup H_{0}\right)} F_{j}(s) d F_{1}(s)
$$

Since by assumption $F_{j}$ is determined by $F$ for all $j \in S$, the integrand is determined by $F$. Also, the integrand is clearly strictly positive, and hence $F_{1}$ can be obtained from this equation. Finally, by replacing $S$ by $S \cup\{1\}$ we can prove identifiability of $(E, \phi)$ by the proof of corollary 2 part (b). 
Note that if $P=\{1\}$, the hypothesis is vacuously satisfied, and the conclusion is that the system is identifiable if the components of $S$ are. For instance, this applies to the system $(E, \phi)$ with $E=\{1,2,3,4\}, \phi(\mathbf{x})=$ $\max \left\{x_{1}, x_{2} x_{3} x_{4}\right\}$.

Theorem 2 Let $(E, \phi)$ be a coherent system with the components in $P=$ $\{1, \ldots, k\}$ in parallel with the rest of the system. Suppose that the system $(S, \psi)$ defined by $S=E-P, \psi\left(x_{k+1}, \ldots, x_{n}\right)=\phi\left(0, \ldots, 0, x_{k+1}, \ldots, x_{n}\right)$ is identifiable. If $P=R_{0} \cup M_{0}$, and $P\left(\cap_{l \in M_{0}}\left(\tau_{l} \leq T\right)\right)>0$, then $(E, \phi)$ is identifiable.

Proof: Put $B=\cap_{l \in M_{0}}\left(\tau_{l} \leq T\right)$. By hypothesis, $B$ has positive probability. Furthermore, $B$ is observed if it occurs. Therefore, $G(t)=P\left(B \cap\left(\max \left\{T_{j} \mid\right.\right.\right.$ $j \in P\})<t)$ is determined by $F$. Let $A_{i}$ be a fatal set of $(S, \psi)$. We then also have that $G_{0}(t)=P\left(B \cap\left(\max \left\{T_{j} \mid j \in P\right\}<T\right) \cap\left(I=A_{i} \cup P\right) \cap(T \leq t)\right.$ is determined by $F$. We then have

$$
G_{0}(t)=\int_{0}^{t} G(s) d G_{i}(s)
$$

where $G_{i}$ is as in (1), referring to the system $(S, \psi)$. Thus, $G_{i}$ is determined by $F$. Since $i$ was arbitrary, all $G_{i}$ 's corresponding to different fatal sets are determined. But this is precisely the information needed to determine $F_{l}$ for each $l \in S$, by the identifiability of $(S, \psi)$. Now we can argue as in corollary 2 part (b) to see that $F_{l}$ is determined by $F$ for $l \in P$. Hence $(E, \phi)$ is identifiable.

For instance, the system $(E, \phi)$ defined by $E=\{1,2,3\}$ and $\phi(\mathbf{x})=$ $\max \left\{x_{1}, x_{2} x_{3}\right\}$ is identifiable if component 1 is lifemonitored.

Corollary 3 Let $E, \phi, P$ be as in theorem 2, and suppose $P\left(\cap_{l \in M_{0}}\left(\tau_{l} \leq\right.\right.$ $T))>0$. Suppose in addition that $S=E-P$ contains at most one component in parallel with the rest of the system, and that $T_{l}$ is exponentially distributed for each $l \in S$. Then $(E, \phi)$ is identifiable.

Proof: By theorem 2 of Antoine et al. (1993) the fatal matrix of $(S, \psi)$ (where $\psi$ is defined as in theorem 2) has full rank, and by theorem 6 of the same paper, $(S, \psi)$ is identifiable. Hence, the corollary follows from theorem 2 .

\section{Identifiability with analytic distribution functions}

As stated in the introduction, Antoine et al. (1993) gives in theorem 4 a sufficient condition for identifiability, assuming analytic distribution functions. 
The main merits of their result are that, at the cost of making this restrictive distributional assumption, they are able to reprove the main theorem of Meilijson (1981) in a much more elementary way (theorem 5 of Antoine et al. (1993)), and that as a consequence of their proof, they can give the strongest possible identifiability result when restricting to certain parametric classes of distributions. Note that all the results of section 2 can be proved to be valid for analytic distribution functions, which are automatically strictly increasing, only on the basis of their result.

In this section, we show that the results of Antoine et al. (1993) can be pushed a little further. However, we have not been able to find any applications of this that can not be treated by means of the results of section 2.

Throughout the rest of the paper, suppose $F_{j}$ is analytic for each $j \in E$. We can write each $F_{j}$ in the form

$$
F_{j}(t)=a_{j 0} t^{r_{j}}\left(1+\sum_{p=1}^{\infty}\left(a_{j p} t^{p}\right)\right)
$$

Let $B$ be the set of components that are known to be identifiable without using rank properties of the minimal, critical or fatal incidence matrices; i.e. $B$ includes $J$ (the components satisfying (2)) and $R_{0}$ (the lifemonitored components); and also the set of $i \in M_{0}$ (the set of conditionally monitored components) satisfying the condition of proposition 1. Put $A=E-B$. For any $\mathbf{x} \in R^{n}$, let $\mathbf{x}_{A}$ and $\mathbf{x}_{B}$ be the vectors with components $x_{i}, i \in A, x_{j}, j \in B$ respectively.

Theorem 3 The system is identifiable if for any diagonal $m \times m$ matrix $R$ whose diagonal entries are positive integers, and for each non-negative integer $p$, the matrix $R D_{A}+p C_{A}$ has full rank (i.e. equal to the cardinality of $A$ ).

Proof: Clearly, for each $i \in\{1, \ldots, m\}$ the function $G_{i}^{\prime}=g_{i}$ (the density function corresponding to the $i$ th fatal set, cf. (1)) is analytic. As in eq. 11 of Antoine et al. (1993) we write $g_{i}$ in the form

$$
g_{i}(t)=b_{i 0} t^{d_{i}}\left(1+\sum_{p=1}^{\infty}\left(b_{i p} t^{p}\right)\right)
$$

In the proof of theorem 4 in that paper it is shown that $D \mathbf{r}=\mathbf{d}+\mathbf{1}$. Here, $\mathbf{r}=$ $\left(r_{1}, \ldots, r_{n}\right), \mathbf{d}=\left(d_{1}, \ldots, d_{m}\right), \mathbf{1}=(1, \ldots, 1)$. Hence, $D_{A} \mathbf{r}_{A}=\mathbf{d}+\mathbf{1}-D_{B} \mathbf{r}_{B}$. Since $r_{j}$ is determined by $F$ for $j \in B$, it follows that $\mathbf{r}$ is determined by $F$ if $D_{A}$ has full rank. Put $\mathbf{a}_{p}=\left(a_{1 p}, \ldots, a_{n p}\right), p=0,1,2, \ldots$. Applying the same argument as above to eq. 15 in Antoine et al. (1993), i.e. to the equation 
$D\left(\log \left(\mathbf{a}_{0}\right)\right)=\left(b_{1}, \ldots, b_{m}\right)$, where $b_{i}=\left(\log \left(b_{i 0}\right) /\left(\sum_{j=1}^{n}\left(r_{j} c_{i j}\right)\right)\right.$ (recall that the $c_{i j}$ 's are the entries of $C$ ), it follows that $\mathbf{a}_{0}$ is determined by $F$ if $D_{A}$ has full rank. Let $R$ be the diagonal matrix with diagonal entries $r_{i i}=\sum_{j=1}^{n}\left(r_{j} c_{i j}\right)$. Antoine et al. (1993) prove that the vector $\mathbf{c}_{p}=(R D+p C) \mathbf{a}_{p}$ is determined by $F$ together with the coefficients $a_{j l}, l<p$ and the integers $r_{j}, j=1, \ldots, n$. If in addition $a_{j p}$ is known for $j \in B, \mathbf{a}_{p}$ can be determined if $R D_{A}+p C_{A}$ has full rank. Hence the theorem follows by induction.

Theorem 4 Suppose that for each $j \in A, F_{j}$ is known to be of one of the following two forms:

(a) $F_{j}(t)=F_{j}\left(t ; \theta_{j}\right)$, where either $a_{j}$ or $r_{j}$ is a one-to-one function of $\theta_{j}$

(b) $F_{j}(t)=F_{j}\left(t ; \alpha_{j}, \beta_{j}\right)$, where $\left(a_{j}, r_{j}\right)$ is a one-to-one function of $\left(\alpha_{j}, \beta_{j}\right)$

Then the system is identifiable if $D_{A}$ has full rank.

Proof: By the proof of theorem $3, a_{j}$ and $r_{j}$ are determined by $F$ if $D_{A}$ has full rank, and the theorem follows.

As pointed out in connection with the analogous theorems 6 and 7 in Antoine et al. (1993), the class of distributions satisfying the hypothesis of the theorem includes the exponential and half-normal distributions, and the Weibull and gamma distributions with integer shape parameters. To illustrate how theorem 4 can be used, we give an alternative proof of a special case of corollary 3 .

Corollary 4 Suppose that the components $\{1, \ldots, k\}$ are in parallel with the rest of the system. Define an observation plan by $R_{0}=\{1\}$ and $H(\emptyset$, $\{1, \ldots, j\})=\{j+1\}$ for $j \leq k-2$ (cf. corollary 2 part (a)). Suppose $T_{j}$ is exponentially distributed for $j=k, \ldots, n$. Then the system is identifiable.

Proof: According to the described observation plan, $R_{0} \cup M_{0}=\{1, \ldots, k-1\}$. Hence by corollary $2 A \subset A^{\prime}=\{k, \ldots, n\}$. The components in $A^{\prime}$ form a subsystem (module) with only one component in parallel with the rest of the system. The corresponding fatal incidence matrix coincides with $D_{A^{\prime}}$, and has full rank by theorem 2 of Antoine et al. (1993). Hence $D_{A}$ has full rank, and the corollary follows from theorem 4.

\section{Acknowledgement}

I am very greatful to Professor Bent Natvig for his valuable comments and numerous suggestions for improvements of the exposition, as well as for his help in proofreading of the manuscript. 


\section{References}

ANTOINE, R., DOSS, H. AND HOLLANDER, M. (1993) On identifiability in the autopsy model of reliability theory. J. Appl. Prob. 30, 913-930.

GÅSEMYR, J. AND NATVIG, B. (1994) Bayesian estimation of component lifetimes based on autopsy data. Tech. report, Department of Mathematics, Univ. of Oslo.

MEILIJSON, I. (1981) Estimation of the lifetime distribution of the parts from the autopsy statistics of the machine. J. Appl. Prob. 18, 829-838.

MEILIJSON, I. (1994) Competing risks on coherent reliability systems. Estimation in the parametric case. J. Amer. Statist. Ass. 89, 1459-1464.

NOWIK, S. (1990) Identifiability problems in coherent systems. J. Appl. Prob. 28, 862-872. 